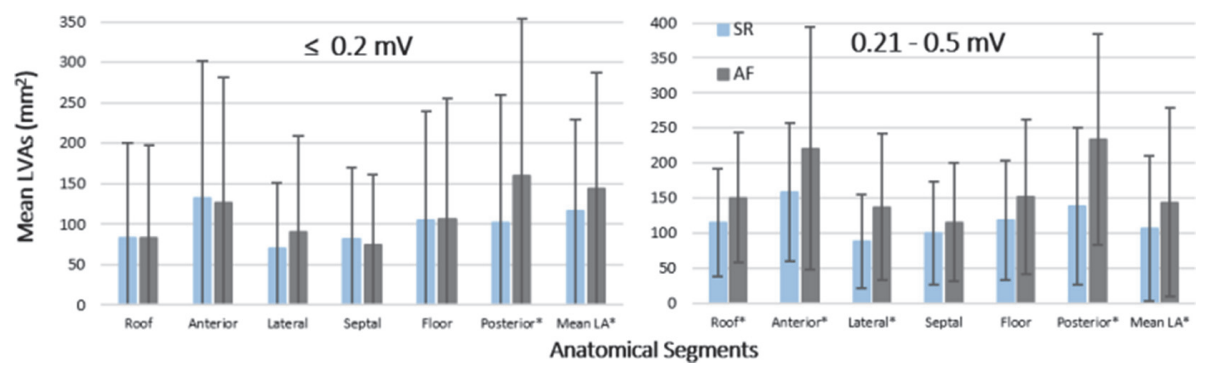

Abstract 2 Figure 2 Bar chart comparing LVA burden in both SR and AF

Figure 2: Bar chart representing the absolute values of low voltage areas $\left(\mathrm{mm}^{2}\right)$ mapped in each anatomical segment indicating a remarkable difference in Posterior LA wall compared to other anatomical segments. The left graph demonstrates Dense Scar and the right graph Diseased LA Tissue as per predefined voltage criteria. *Denotes significance. LVA=Low voltage area; $L A=$ Left Atrium; SR=Sinus Rhythm; $A F=A$ trial Fibrillation.

was observed on the posterior wall, once again with the highest correlation of all segments $(\mathrm{R}=0.314, \mathrm{p}=0.015)$.

Conclusions The automated VHA tool demonstrated the posterior wall as the only anatomical segment to be significantly higher in LVAs consistent with dense scar burden (i.e. $\leq 0.2 \mathrm{mV}$ ), when mapped in AF. Mapping rhythm has a substantial impact on surrogate readings of both 'Diseased Tissue' $0.21-0.5 \mathrm{mV}$ and 'Dense Scar' $\leq 0.2 \mathrm{mV}$ on the posterior wall. These findings mandate careful interrogation of posterior wall voltages and may partially explain the debated outcome for posterior wall ablation. It is best interrogated in both rhythms to confirm the presence of arrhythmogenic substrates over artifactitious rhythm effect.

\section{ATRIAL UPTAKE ON TECHNETIUM PYROPHOSPHATE SCINTIGRAPHY: CLINICAL IMPLICATIONS OF THIS NEW PHENOMENON}

${ }^{1,2} \mathrm{M}$ Hussain, ${ }^{2} \mathrm{M}$ Hanna, ${ }^{2} \mathrm{E}$ Donnellan, ${ }^{2} \mathrm{~A}$ Krywanczyk, ${ }^{2} \mathrm{O}$ Abou Hassan, ${ }^{2} \mathrm{~T}$ Martyn, ${ }^{2} \mathrm{~S}$ Alkharabsheh, ${ }^{1,2} \mathrm{C}$ Watson, ${ }^{2} \mathrm{C}$ Tan, ${ }^{2} \mathrm{ER}$ Rodriguez, ${ }^{2} \mathrm{WH}$ Wilson Tang, ${ }^{2} \mathrm{D}$ Kwon, ${ }^{2} \mathrm{P}$ Cremer, ${ }^{2} \mathrm{~B}$ Griffin, ${ }^{2} \mathrm{~F}$ Cheng, ${ }^{2} \mathrm{M}$ Kanj, ${ }^{2} \mathrm{~W}$ Jaber, ${ }^{1,2} \mathrm{P}$ Collier. ${ }^{1}$ Queen's University Belfast, UK: ${ }^{2}$ Cleveland Clinic, USA

10.1136/heartjnl-2021-ICS.3
Transthyretin cardiac amyloidosis (ATTR-CA) is an increasingly recognized disease in which atrial fibrillation $(\mathrm{AF})$ has been shown to be highly prevalent. $99 \mathrm{~m}$-Technetium pyrophosphate (99mTc-PyP) scanning is used to non-invasively make the diagnosis of ATTR-CA. Assessment of atrial wall uptake (AU) on $99 \mathrm{mTc}-\mathrm{PyP}$ is currently not utilized in the clinical setting. We hypothesize that AU by $99 \mathrm{mTc}-\mathrm{PyP}$ is associated with higher rates of $\mathrm{AF}$, and $\mathrm{AU}$ corresponds to atrial wall infiltration by TTR fibril deposition. All patients that underwent consecutive 99mTc-PyP at our institution for suspected ATTR-CA from January 2012 to September 2019 were included. Presence or absence of AU was assessed visually using Corridor 4DM software (v2015) using fused/combination computed tomographysingle photon emission computed tomography (SPECT) imaging in all cases (figure 1). ATTR-CA diagnosis was defined using institutional guidelines. Patient characteristics were obtained through electronic medical records. Retrospective review of the patient population identified those who had left atrial tissue available from heart transplantation, left atrial resection or autopsy to allow for direct tissue-imaging correlation (figure 1). A total cohort of 580 patients were identified (table 1). 164 (28\%) patients had scans consistent with ATTRCA. 296 (51\%) had a diagnosis of AF. 117 (20\%) patients had AU. Table 2 shows the relative interaction of AU, ATTR-

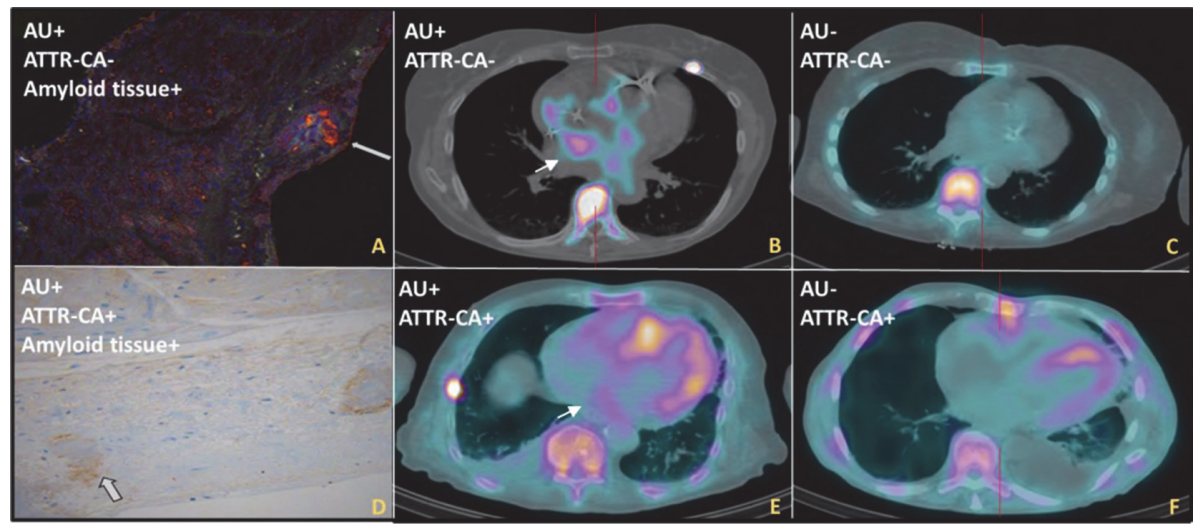

Abstract 3 Figure $199 \mathrm{~m}$-Technetium pyrophosphate scan (99mTc-PyP) illustrating atrial uptake (AU) in patients with/without a clinical diagnosis of transthyretin cardiac amyloidosis (ATTR-CA) with histopathological correlation.

Caption:

A: Left atrial appendage resection specimen in a patient AU+, ATTR-CA- with Congo red stain (40x) showing apple-green and orange birefringence (arrow), confirming the material is amyloid.

B: 99mTc-PyP scan showing AU+ in a patient ATTR-CA-.

C: $99 m T c-P y P$ scan showing AU- in a patient ATTR-CA-

D: Left atrial appendage resection specimen in a patient AU+, ATTR-CA+ with immunohistochemistry positive for TTR (20x). Brown staining visible in the amyloid nodules (arrow).

E: 99mTc-PyP scan showing $\mathrm{AU}+$ in a patient ATTR-CA+.

F: 99mTc-PyP scan showing AU- in a patient ATTR-CA+. 
Abstract 3 Table 1 Baseline patient characteristics at the time of 99mTc-PvP scan

Caption: Data is provided for the total cohort; for those with and without a clinical diagnosis of transthyretin cardiac amyloidosis; for those with and without a clinical diagnosis of atrial fibrillation (AF): and for those with and without atrial uptake on 99mTc-PvP scan respectively.

\begin{tabular}{|c|c|c|c|c|c|c|c|c|c|c|}
\hline Characteristic & $\begin{array}{l}\begin{array}{c}\text { Total } \\
\text { Cohort }\end{array} \\
\mathrm{N}=580\end{array}$ & $\begin{array}{c}\text { Am } \\
+ \\
\mathrm{n}=164\end{array}$ & $\begin{array}{c}\text { loid } \\
n=416\end{array}$ & $p$ value & $\begin{array}{c}+ \\
n=296\end{array}$ & $\mathrm{n}=284$ & $p$ value & $\begin{array}{l}\text { Atrial } \\
+ \\
\mathrm{n}=117\end{array}$ & $\begin{array}{l}\text { Jptake } \\
- \\
n=463\end{array}$ & p value \\
\hline $\begin{array}{l}\text { Median age, } \\
\text { years (IQR) }\end{array}$ & $\begin{array}{c}74 \\
(17)\end{array}$ & $\begin{array}{c}79 \\
(11)\end{array}$ & $\begin{array}{c}72 \\
(18)\end{array}$ & $p<0.001$ & $\begin{array}{c}76 \\
(14)\end{array}$ & $\begin{array}{c}70 \\
(19)\end{array}$ & $p<0.001$ & $\begin{array}{c}77 \\
(12)\end{array}$ & $\begin{array}{c}72 \\
(18)\end{array}$ & $p<0.001$ \\
\hline \multicolumn{11}{|l|}{ Gender } \\
\hline Male & $\begin{array}{c}398 \\
(69 \%)\end{array}$ & $\begin{array}{c}135 \\
(23 \%)\end{array}$ & $\begin{array}{c}263 \\
(45 \%)\end{array}$ & $p<0.001$ & $\begin{array}{c}203 \\
(35 \%)\end{array}$ & $\begin{array}{c}195 \\
(34 \%)\end{array}$ & $p=1.00$ & $\begin{array}{c}81 \\
(14 \%)\end{array}$ & $\begin{array}{c}317 \\
(55 \%)\end{array}$ & $p=0.84$ \\
\hline Female & $\begin{array}{c}182 \\
(31 \%)\end{array}$ & $\begin{array}{c}29 \\
(5 \%)\end{array}$ & $\begin{array}{c}153 \\
(26 \%) \\
\end{array}$ & $p<0.001$ & $\begin{array}{c}93 \\
(16 \%) \\
\end{array}$ & $\begin{array}{c}89 \\
(15 \%)\end{array}$ & $p=1.00$ & $\begin{array}{c}36 \\
(6 \%) \\
\end{array}$ & $\begin{array}{c}146 \\
(25 \%)\end{array}$ & $p=0.85$ \\
\hline \multicolumn{11}{|l|}{ Race } \\
\hline White & $\begin{array}{c}377 \\
(65 \%)\end{array}$ & $\begin{array}{c}109 \\
(19 \%)\end{array}$ & $\begin{array}{c}268 \\
(46 \%)\end{array}$ & $p=0.50$ & $\begin{array}{c}220 \\
(38 \%)\end{array}$ & $\begin{array}{c}157 \\
(27 \%)\end{array}$ & $p<0.001$ & $\begin{array}{c}84 \\
(14 \%)\end{array}$ & $\begin{array}{c}293 \\
(50 \%)\end{array}$ & $p=0.11$ \\
\hline Black & $\begin{array}{c}188 \\
(32 \%)\end{array}$ & $\begin{array}{c}54 \\
(9 \%)\end{array}$ & $\begin{array}{c}134 \\
(23 \%)\end{array}$ & $p=1.00$ & $\begin{array}{c}72 \\
(12 \%)\end{array}$ & $\begin{array}{c}116 \\
(20 \%)\end{array}$ & $p<0.001$ & $\begin{array}{c}33 \\
(6 \%)\end{array}$ & $\begin{array}{c}155 \\
(27 \%)\end{array}$ & $p=0.41$ \\
\hline Other & $\begin{array}{c}15 \\
(3 \%)\end{array}$ & $\begin{array}{c}1 \\
(<1 \%)\end{array}$ & $\begin{array}{c}14 \\
(2 \%)\end{array}$ & $\mathrm{p}=0.08$ & $\begin{array}{c}4 \\
(<1 \%)\end{array}$ & $\begin{array}{c}11 \\
(2 \%)\end{array}$ & $p=0.020$ & $\begin{array}{c}0 \\
(0 \%)\end{array}$ & $\begin{array}{c}15 \\
(3 \%)\end{array}$ & $p=0.06$ \\
\hline \multicolumn{11}{|l|}{$\begin{array}{l}\text { Body mass } \\
\text { index }\left(\mathrm{kg} / \mathrm{m}^{2}\right)\end{array}$} \\
\hline$>30$ & $\begin{array}{c}215 \\
(37 \%)\end{array}$ & $\begin{array}{c}46 \\
(8 \%)\end{array}$ & $\begin{array}{c}169 \\
(29 \%)\end{array}$ & $p=0.004$ & $\begin{array}{c}103 \\
(18 \%)\end{array}$ & $\begin{array}{c}112 \\
(19 \%)\end{array}$ & $\mathrm{p}=0.32$ & $\begin{array}{c}35 \\
(6 \%)\end{array}$ & $\begin{array}{c}180 \\
(31 \%)\end{array}$ & $p=0.07$ \\
\hline $25-30$ & $\begin{array}{c}220 \\
(38 \%)\end{array}$ & $\begin{array}{c}73 \\
(13 \%)\end{array}$ & $\begin{array}{c}147 \\
(25 \%)\end{array}$ & $\mathrm{p}=0.015$ & $\begin{array}{c}125 \\
(22 \%)\end{array}$ & $\begin{array}{c}95 \\
(16 \%)\end{array}$ & $p=0.025$ & $\begin{array}{c}57 \\
(10 \%)\end{array}$ & $\begin{array}{c}163 \\
(28 \%)\end{array}$ & $p=0.005$ \\
\hline$<25$ & $\begin{array}{c}145 \\
(25 \%)\end{array}$ & $\begin{array}{c}45 \\
(8 \%)\end{array}$ & $\begin{array}{c}100 \\
(17 \%)\end{array}$ & $\mathrm{p}=0.32$ & $\begin{array}{c}68 \\
(12 \%)\end{array}$ & $\begin{array}{c}77 \\
(13 \%)\end{array}$ & $p=0.27$ & $\begin{array}{c}25 \\
(4 \%)\end{array}$ & $\begin{array}{c}120 \\
(21 \%)\end{array}$ & $p=0.26$ \\
\hline \multicolumn{11}{|l|}{ CV risk factors } \\
\hline Hypertension & $\begin{array}{c}481 \\
(83 \%)\end{array}$ & $\begin{array}{c}124 \\
(21 \%)\end{array}$ & $\begin{array}{c}357 \\
(62 \%)\end{array}$ & $p=0.004$ & $\begin{array}{c}242 \\
(42 \%)\end{array}$ & $\begin{array}{c}239 \\
(41 \%)\end{array}$ & $p=0.52$ & $\begin{array}{c}90 \\
(16 \%)\end{array}$ & $\begin{array}{c}391 \\
(67 \%)\end{array}$ & $p=0.07$ \\
\hline Hyperlipidemia & $\begin{array}{c}409 \\
(71 \%)\end{array}$ & $\begin{array}{c}120 \\
(21 \%)\end{array}$ & $\begin{array}{c}289 \\
(50 \%)\end{array}$ & $p=0.34$ & $\begin{array}{c}221 \\
(38 \%)\end{array}$ & $\begin{array}{c}188 \\
(32 \%)\end{array}$ & $\mathrm{p}=0.018$ & $\begin{array}{c}90 \\
(16 \%)\end{array}$ & $\begin{array}{c}319 \\
(55 \%)\end{array}$ & $p=0.09$ \\
\hline $\begin{array}{l}\text { Current/ } \\
\text { previous } \\
\text { smoker }\end{array}$ & $\begin{array}{c}328 \\
(57 \%)\end{array}$ & $\begin{array}{c}88 \\
(15 \%)\end{array}$ & $\begin{array}{c}240 \\
(41 \%)\end{array}$ & $p=0.38$ & $\begin{array}{c}169 \\
(29 \%)\end{array}$ & $\begin{array}{c}159 \\
(27 \%)\end{array}$ & $\mathrm{p}=0.81$ & $\begin{array}{c}64 \\
(11 \%)\end{array}$ & $\begin{array}{c}264 \\
(45 \%)\end{array}$ & $p=0.70$ \\
\hline $\begin{array}{l}\text { Diabetes } \\
\text { mellitus }\end{array}$ & $\begin{array}{c}230 \\
(40 \%)\end{array}$ & $\begin{array}{c}40 \\
(7 \%)\end{array}$ & $\begin{array}{c}190 \\
(33 \%)\end{array}$ & $p<0.001$ & $\begin{array}{c}116 \\
(20 \%)\end{array}$ & $\begin{array}{c}114 \\
(20 \%)\end{array}$ & $p=0.81$ & $\begin{array}{c}41 \\
(7 \%)\end{array}$ & $\begin{array}{c}189 \\
(33 \%)\end{array}$ & $p=0.23$ \\
\hline \multicolumn{11}{|l|}{$\begin{array}{l}\text { Labs median, } \\
\text { (IQR) }\end{array}$} \\
\hline Creatinine & $\begin{array}{c}1.33 \\
(0.81)\end{array}$ & $\begin{array}{c}1.34 \\
(0.62)\end{array}$ & $\begin{array}{c}1.31 \\
(0.92)\end{array}$ & $\mathrm{p}=0.84$ & $\begin{array}{c}1.40 \\
(0.95)\end{array}$ & $\begin{array}{c}1.27 \\
(0.73)\end{array}$ & $p=0.07$ & $\begin{array}{c}1.42 \\
(0.74)\end{array}$ & $\begin{array}{c}1.30 \\
(0.81)\end{array}$ & $p=0.84$ \\
\hline eGFR & $\begin{array}{c}40 \\
(21)\end{array}$ & $\begin{array}{c}44 \\
(20)\end{array}$ & $\begin{array}{c}38 \\
(24)\end{array}$ & $p=0.15$ & $\begin{array}{c}40 \\
(21)\end{array}$ & $\begin{array}{c}40 \\
(23)\end{array}$ & $p=1.00$ & $\begin{array}{c}42 \\
\text { (17) }\end{array}$ & $\begin{array}{c}40 \\
(23)\end{array}$ & $p=0.89$ \\
\hline Hemoglobin & $\begin{array}{l}12.3 \\
(3.3)\end{array}$ & $\begin{array}{l}12.8 \\
(2.7)\end{array}$ & $\begin{array}{l}12.2 \\
(3.6)\end{array}$ & $p<0.001$ & $\begin{array}{l}12.1 \\
(3.2)\end{array}$ & $\begin{array}{l}12.6 \\
(3.4)\end{array}$ & $p=0.07$ & $\begin{array}{l}12.3 \\
(2.8)\end{array}$ & $\begin{array}{l}12.4 \\
(3.5)\end{array}$ & $p=0.78$ \\
\hline NT-pro BNP & $\begin{array}{c}2494 \\
(5700)\end{array}$ & $\begin{array}{c}3412 \\
(5363)\end{array}$ & $\begin{array}{c}2040 \\
(5936)\end{array}$ & $p<0.001$ & $\begin{array}{c}3141 \\
(5502)\end{array}$ & $\begin{array}{c}1854 \\
(5986)\end{array}$ & $p=0.008$ & $\begin{array}{c}2799 \\
(4971)\end{array}$ & $\begin{array}{c}2443 \\
(6040)\end{array}$ & $p=0.15$ \\
\hline Troponin T & $\begin{array}{l}0.059 \\
(0.11)\end{array}$ & $\begin{array}{l}0.055 \\
(0.09)\end{array}$ & $\begin{array}{l}0.065 \\
(0.16)\end{array}$ & $p=0.004$ & $\begin{array}{l}0.058 \\
(0.12)\end{array}$ & $\begin{array}{l}0.059 \\
(0.11)\end{array}$ & $p=0.92$ & $\begin{array}{l}0.053 \\
(0.09)\end{array}$ & $\begin{array}{l}0.062 \\
(0.13)\end{array}$ & $p=0.49$ \\
\hline Gilmore stage1 & $\begin{array}{c}222 \\
(38 \%)\end{array}$ & $\begin{array}{c}61 \\
(11 \%)\end{array}$ & $\begin{array}{c}161 \\
(28 \%)\end{array}$ & $p=1.00$ & $\begin{array}{c}101 \\
(17 \%)\end{array}$ & $\begin{array}{c}121 \\
(21 \%)\end{array}$ & $p=0.026$ & $\begin{array}{c}48 \\
(8 \%)\end{array}$ & $\begin{array}{c}174 \\
(30 \%)\end{array}$ & $p=0.55$ \\
\hline Gilmore stage2 & $\begin{array}{c}178 \\
(31 \%)\end{array}$ & $\begin{array}{c}49 \\
(8 \%)\end{array}$ & $\begin{array}{c}129 \\
(22 \%)\end{array}$ & $p=1.00$ & $\begin{array}{c}90 \\
(16 \%)\end{array}$ & $\begin{array}{c}88 \\
(15 \%)\end{array}$ & $\mathrm{p}=0.80$ & $\begin{array}{c}34 \\
(6 \%)\end{array}$ & $\begin{array}{c}144 \\
(25 \%)\end{array}$ & $p=0.67$ \\
\hline Gilmore stage3 & $\begin{array}{c}138 \\
(24 \%)\end{array}$ & $\begin{array}{c}44 \\
(8 \%)\end{array}$ & $\begin{array}{c}94 \\
(16 \%)\end{array}$ & $\mathrm{p}=0.13$ & $\begin{array}{c}85 \\
(15 \%)\end{array}$ & $\begin{array}{c}53 \\
(9 \%)\end{array}$ & $p=0.005$ & $\begin{array}{c}32 \\
(6 \%)\end{array}$ & $\begin{array}{c}106 \\
(18 \%)\end{array}$ & $p=0.36$ \\
\hline $\begin{array}{l}\text { Gilmore stage } \\
\text { unknown }\end{array}$ & $\begin{array}{c}42 \\
(7 \%)\end{array}$ & $\begin{array}{c}5 \\
(<1 \%)\end{array}$ & $\begin{array}{c}37 \\
(6 \%)\end{array}$ & & $\begin{array}{c}21 \\
(4 \%)\end{array}$ & $\begin{array}{c}22 \\
(4 \%)\end{array}$ & & $\begin{array}{c}4 \\
(<1 \%)\end{array}$ & $\begin{array}{c}38 \\
(7 \%)\end{array}$ & \\
\hline \multicolumn{11}{|l|}{ Echo } \\
\hline $\begin{array}{l}\text { LVEF, \% } \\
\text { Median (IQR) }\end{array}$ & $\begin{array}{c}54 \\
(18)\end{array}$ & $\begin{array}{c}51 \\
(17)\end{array}$ & $\begin{array}{c}55 \\
(20)\end{array}$ & $\mathrm{p}=0.025$ & $\begin{array}{c}52 \\
(18)\end{array}$ & $\begin{array}{c}56 \\
(20)\end{array}$ & $p=0.012$ & $\begin{array}{c}50 \\
(20)\end{array}$ & $\begin{array}{c}55 \\
(18)\end{array}$ & $p=0.009$ \\
\hline $\begin{array}{l}\text { Severe mitral } \\
\text { valve disease }\end{array}$ & $\begin{array}{c}8 \\
(1 \%)\end{array}$ & $\begin{array}{c}4 \\
(<1 \%)\end{array}$ & $\begin{array}{c}7 \\
(<1 \%)\end{array}$ & $p=0.47$ & $\begin{array}{c}4 \\
(<1 \%)\end{array}$ & $\begin{array}{c}3 \\
(<1 \%)\end{array}$ & $p=1.00$ & $\begin{array}{c}2 \\
(2 \%)\end{array}$ & $\begin{array}{c}5 \\
(1 \%)\end{array}$ & $p=0.37$ \\
\hline
\end{tabular}


Abstract 3 Table 2 Relative interaction of atrial update, transthyretin cardiac amyloidosis (ATTR-CA) and atrial fibrillation (AF). Caption: Total incudes total number of patients between the 2 groups

\begin{tabular}{|c|c|c|c|c|c|c|c|c|}
\hline \multirow{2}{*}{$\begin{array}{c}\text { Total Cohort } \\
\text { N=580 }\end{array}$} & \multicolumn{5}{|c|}{ Atrial Uptake(+) } & \multicolumn{4}{c|}{ Atrial Uptake(-) } \\
\hline ATTR-CA(-) & 58 & $10 \%$ & 51 & $9 \%$ & 358 & $62 \%$ & 146 & $25 \%$ \\
\hline ATTR-CA(+) & 59 & $10 \%$ & 56 & $10 \%$ & 105 & $18 \%$ & 43 & $7 \%$ \\
\hline
\end{tabular}

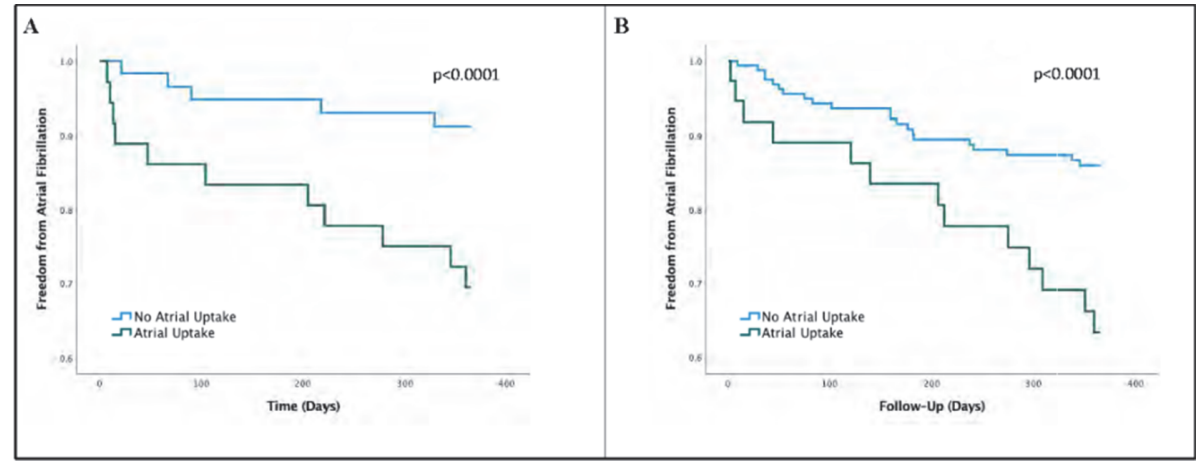

Abstract 3 Figure 2 Freedom from atrial fibrillation in patients with and without atrial uptake

CA and AF. Of 117 patients with AU, 107 (91\%) had AF. In contrast, of 463 patients without AU 189 (41\%) had AF. AU was associated with a significantly higher incidence of $\mathrm{AF}$ in those with and without ATTR-CA $(\mathrm{p}<0.001)$. On Cox proportional hazards analyses, freedom from $\mathrm{AF}$ at 1-year in patients with ATTR-CA was significantly lower in patients with AU (HR: 2.27; 95\% CI: 1.37-3.78; p<0.0001) (figure 2). Similarly, freedom from $\mathrm{AF}$ at 1 -year in patients without ATTR-CA was significantly lower in patients with AU (HR: 2.21; 95\% CI: 1.46-3.34; p<0.0001). On Kaplan-Meier survival analysis, there were no significant differences in mortality rates in patients with AF and ATTR-CA; with AU vs. without AU (all-cause mortality, $\mathrm{p}=0.79$; 1-year mortality, $\mathrm{p}=0.76$ ). There were no significant differences in mortality rates in patients with AF and without ATTR-CA; with AU vs. without AU (all-cause mortality, $p=0.20 ; 1$-year mortality, $p=0.57$ ). For the first time, we described that the presence of $\mathrm{AU}$ was independent of ATTR-CA diagnosis and was associated with higher rates of AF. AU was not associated with increased mortality in those with AF. This leads to the question of the role of $\mathrm{AU}$ in $\mathrm{AF}$ diagnosis/management and the interaction of $\mathrm{AU}$ with ATTR-CA diagnosis.

\section{PREDICTIVE GENETIC TESTING IN INHERITED CARDIAC CONDITIONS: FINDINGS FROM A LARGE IRISH COHORT}

1J Murphy, ${ }^{1} \mathrm{C}$ Kirk, ${ }^{2} \mathrm{G}$ Abboud Guerra, ${ }^{3} \mathrm{~J}$ Galvin, ${ }^{4} \mathrm{D}$ Ward, ${ }^{5} \mathrm{~T}$ Prendiville, ${ }^{3} \mathrm{C}$ McGorrian, ${ }^{5}$ SA Lynch. 'University College Dublin, Ireland; ${ }^{2}$ St Vincent's University Hospital, Ireland; ${ }^{3}$ Mater University Hospital, Ireland; ${ }^{4}$ Tallaght University Hospital, Dublin, Ireland; ${ }^{5}$ Children's Health Ireland Crumlin, Dublin, Ireland

10.1136/heartjnl-2021-ICS.4
Introduction Inherited cardiac conditions comprising cardiomyopathies (hypertrophic, dilated, arrhythmogenic) and cardiac ion channelopathies (long QT/Brugada syndromes, CPVT) predispose to sudden cardiac death. Predictive genetic testing of at-risk family members informs patient management and is cost-effective as those who test negative can be discharged from lifelong cardiac follow-up. Aim: Through predictive testing uptake, investigate the demographics and genetic contribution of inherited cardiac conditions in the Republic of Ireland. Methods Predictive testing data from 2003 to 2020 was collated through interrogation of departmental databases and molecular genetic reports at a national genetics centre and two tertiary cardiac referral clinics.

Results Collectively, 1,535 predictive tests for pathogenic/likely pathogenic variants were undertaken in 1,508 individuals from 241 families, including 27 individuals tested for two familial variants. The median age at testing was 36 years (range $0.07-$ 90 years). From 1,152 adults, more females (58\%) presented for testing than males (42\%). Amongst 365 children, marginally more boys (53\%) were tested than girls (47\%). On average, six individuals per family were tested (range 1 - 84). Seven hundred and nineteen individuals (48\%) were found to be genotype-positive for their familial variant(s). Overall, predictive testing was performed for 26 genes. Testing for long QT syndrome (LQTS) and hypertrophic cardiomyopathy (HCM) genes represented $48 \%$ and $29 \%$ of the cohort, respectively. Other cardiomyopathies and arrhythmia syndromes accounted for $10 \%$ and $8 \%$ respectively, with $5 \%$ tested for syndromic disease genes. The most frequently tested gene was KCNQ1 (LQTS; 24\% of tests), followed by MYBPC3 (HCM/DCM; 20\%), KCNH2 (LQTS; 17\%), MYH7 (HCM/DCM; 8\%) and SCN5A (LQT/Brugada syndromes; $8 \%)$. There was an absence of testing for several sarcomeric 